\title{
Pulmonary clearance of UICC amosite fibres inhaled by rats during chronic exposure at low concentration
}

\author{
A D JONES, C H McMillan, A M JOHNSTON, C McINTOSH, H COWIE, \\ R E BOLTON, G BORZUCKI, J H VINCENT
}

From the Institute of Occupational Medicine, Edinburgh EH8 9SU, UK

ABSTRACT Clearance of UICC amosite asbestos from the lungs during chronic - that is, repeatedexposure was investigated by using the scanning electron microscope to measure lung burdens from rats which had inhaled amosite asbestos at an approximately constant concentration of $0.1 \mathrm{mg} / \mathrm{m}^{3} \mathrm{or}$, equivalently, $20 \mathrm{fibres} / \mathrm{ml}$ for seven hours a day, five days a week for up to 18 months. The lung burdens were compared with previous results for higher exposure concentrations of $1 \mathrm{and} 10 \mathrm{mg} / \mathrm{m}^{3}$. Those previous lung burdens had been measured using other analytical methods (infrared spectrophotometry) that were not suitable for the new lower lung burdens. Taken together, these results showed lung burdens rising pro rata with exposure concentration and exposure time. This accumulation of lung burden has been described by a kinetic model that takes account of the sequestration of material at locations in the lung from where it cannot be cleared. Unlike some earlier models in which lung burdens eventually reach a plateau with equilibrium between deposition and clearance during chronic exposure, this sequestration model shows lung burdens continuing to rise with exposure time. The latest results reported here support the application of such a model to lower exposure concentrations closer to those of asbestos in workplaces.

The amount of material retained by the lung during and after exposure to airborne dusts is determined by the physical processes governing inhalation and deposition and the biological processes governing clearance. In some circumstances the dust may be inhaled on a single occasion (which is often the concern with, for example, radioactive aerosols) and the amount of material retained (at any subsequent time) may be estimated from mathematical models describing the clearance of particles from the lung. More typically, however, in most occupational settings the dust may be received as a prolonged series of repeated exposures (over a working lifetime). The mathematical model that describes deposition and clearance for a single exposure should, in principle, also be sufficient to describe the accumulation of material during chronic exposure, provided that the performance of the lung clearance mechanisms remains unchanged. Middleton $e t$ al, however, found that the application of a simple model with coefficients derived from data for clearance of amosite asbestos from lungs of rats exposed for short periods (up to six weeks) led to an underestimate of the amount of dust

Accepted 11 May 1987 retained after a year of exposure to the same dust. ${ }^{12}$ Several other studies dealing with various inhaled particles suggested that the rate of clearance of dust after a short period of exposure depends on the amount deposited in the lung. ${ }^{3-5}$ More precisely, the normalised rate of clearance $(R$, the amount cleared per unit time at time $t /$ the lung burden at time $t$ ) was considerably lower for lung burdens $B$ above some critical level. These findings formed the basis for the adoption of a clearance overload hypothesis ${ }^{6}$ for lung clearance during continuing chronic exposure.

In a previous paper we reported the results of an experiment in which two groups of rats were exposed to amosite asbestos at two respirable concentrations (1 and $10 \mathrm{mg} / \mathrm{m}^{3}$ ) for periods up to one year. ${ }^{7}$ Under the overload hypothesis it was expected that, for the lower concentration, the lung burden would not reach the critical level at which clearance mechanisms were expected to be overloaded. Moreover, for the lower concentration, it was expected that the rates of deposition $D_{L}$ into, and clearance from, the lung would eventually reach equilibrium and the lung burden $B_{L}$ would reach a plateau during continued chronic exposure at constant concentration. When this occurs, 


$$
\mathbf{R B}_{\mathrm{L}}=\mathrm{D}_{\mathrm{L}}
$$

For the higher concentration, it was expected that the lung burden $B_{H}$ would reach the critical level for clearance mechanisms to be overloaded and that consequently the normalised clearance rate $\mathrm{R}$ would diminish (to $R_{H}$ ) such that the clearance rate would always be exceeded by the deposition rate $D_{H}$, that is

$$
\mathbf{R}_{\mathrm{H}} \mathbf{B}_{\mathrm{H}}<\mathrm{D}_{\mathrm{H}}
$$

In this case the lung burden $B_{H}$ should continue to increase throughout the exposure period.

The results from the experiment, however, showed that the lung burdens continued to increase pro rata with exposure time and exposure concentration for both groups, without any sign of the lung burdens reaching a plateau. ${ }^{7}$ The apparent absence of any dependence of clearance on the lung burden throughout the chronic exposure led to the proposal to include a sequestration compartment (as suggested by Chan $e t$ $a l^{8}$ ) in the mathematical model of clearance. ${ }^{7}$ This sequestration compartment was designed to take account of some fraction of the deposited material becoming sequestered in parts of the lung from which clearance is negligible. The modified model satisfactorily described the experimental results which showed the lung burden increasing in almost linear proportion to the time of exposure and in direct proportion to the respirable dust concentration.

The inclusion of a sequestration process in the model of the deposition and clearance kinetics of inhaled particles has considerable implications for occupational epidemiology, since any examination of data for the relation between dust exposure, dose received by the lung, and the incidence of respiratory disease will be influenced by the choice of model of deposition and clearance. Exercises of this type have already been conducted by Smith using a model in which clearance rates during chronic exposure were dependent on concentration ${ }^{9}$ and Rappaport using a model in which the lung burden approaches a plateau. ${ }^{10}$ The sequestration model, by contrast, predicts neither the concentration dependence for lung clearance nor a plateau for the lung burden during the chronic exposure, but rather a lung burden increasing approximately pro rata with cumulative exposure.

Our earlier results on chronic exposure which formed the principal basis for the sequestration model $^{7}$ were obtained at two airborne dust concentrations of UICC amosite asbestos, 1 and $10 \mathrm{mg} / \mathrm{g}^{3}$. It is clearly desirable to widen the span of concentrations against which to test a model predicting proportionality of lung burden on concentration, and particularly valuable to do this with a low concentration where clearance overload would not be expected. In the present paper we report the results of a further study of chronic exposure that extends the range of airborne concentrations downwards by another decade to a target concentration of $0.1 \mathrm{mg} / \mathrm{m}^{3}$ (equivalent to 15 fibres $/ \mathrm{ml}$ for fibres longer than $5 \mu \mathrm{m}$ ). The aim is to examine the extent to which ideas developed at higher concentrations may be applied at much lower ones that are more akin to exposure to asbestos at the workplace.

\section{Methods}

The experiment was conducted using outbred male SPF Wistar rats of the AF/HAN strain, exposed to airborne dust of UICC amosite in a $1 \mathrm{~m}^{3}$ inhalation chamber in separate groups of 12 animals (with up to 48 in the chamber at any given time). The details of the inhalation chambers have been described elsewhere." The amosite asbestos was dispersed into the inhalation chamber using a rotating table air venturi dust dispenser which (for reasons of safety) was enclosed in a dust tight container with a high efficiency filter on the air entry. The required constant daily average dust concentration was obtained by placing at the start of each day a fixed amount, $100 \mathrm{mg}$, of amosite asbestos on the rotating table which was geared to rotate just once during the daily exposure period. The dusting regimen and times of insertion into the chamber of individual rats were chosen so that the rats could be exposed for durations of up to 20 months (for seven hours a day on five days a week). Dust exposure levels were defined in terms of the average respirable dust concentrations as measured using Casella Type MRE $113 \mathrm{~A}$ gravimetric dust samplers. Each dust sample was collected over five days of dusting and with, as a check, two samplers sampling simultaneously. The samples were taken on vinyl metricel (VM1) filters, and evaluated both gravimetrically and using on-filter infrared absorption spectroscopy to assess the amount of asbestos in each dust sample. Thus account was taken of the presence of background dust arising from the animal food and the activity of the animals. This background respirable dust concentration was of the order of $0.02 \mathrm{mg} / \mathrm{m}^{3}$ (an amount that had been trivial in all previous experiments conducted at higher test dust concentrations).

The number concentration of airborne fibres in the exposure chamber was determined by sampling, with an open face (Gelman) sampler head facing downwards, at a sampling flowrate of $50 \mathrm{ml} / \mathrm{min}$. These samples were collected on membrane filters over full seven hour periods of dusting; the fibres on the filter were counted using phase contrast optical microscopy and standard counting procedures. ${ }^{12}$

Animals were removed in groups of 12 after preselected exposure times ( $\mathrm{T}$ ) and killed in subgroups of six at postexposure times $(\triangle T)$ of three and 38 days. After the lungs (including the associated mediastinal 
and hilar lymph nodes) were excised in toto, the burdens of dust were recovered using the low temperature plasma ashing method described elsewhere. ${ }^{6}$ In the previous experiments of this type conducted at higher dust concentrations the lung burdens had been assayed using the infrared spectrophotometric method. This analytical method, however, was found to be not sensitive enough to allow detection of the lung burdens from the exposure at the target level of $0.1 \mathrm{mg} / \mathrm{m}^{3}$. Instead, therefore, an electron microscope technique was used. In this technique the ashed lung residue was washed into a plastic bottle with $5 \mathrm{ml}$ of $0.2 \mathrm{M} \mathrm{HCL}$ and $15 \mathrm{ml}$ of filtered, distilled water and then ultrasonicated for one minute. Next, an aliquot was taken, washed in dilute acid, and the fibres collected on a $0.2 \mu \mathrm{m}$ pore size Nuclepore filter. Then the filter was mounted on an electron microscope specimen stub using conductive colloidal graphite and coated with a layer of gold. The fibres were counted and sized using the scanning electron microscope at magnifications of $10000 \times$ and $4000 \times$ for fibres longer than $0.4 \mu \mathrm{m}$ and for fibres longer than $5 \mu \mathrm{m}$ respectively. This two stage counting and sizing procedure was adopted both to provide data for as wide a range of sizes as possible and to direct the greater part of the counting effort to the part of the size range (longer than $5 \mu \mathrm{m}$ ) which contained the greater proportion $(-70 \%)$ of the mass. The volume of the fibres was calculated from the width and length measurements using a shape factor determined by Burdett, ${ }^{13}$ and this volume was converted to mass using the density of UICC amosite $\left(3.6 \times 10^{3} \mathrm{~kg} / \mathrm{m}^{3}\right)$. The validity of this conversion process was confirmed by assessing a sample laden with a known amount of asbestos.

\section{Results and conclusions}

The dust recovery procedures were designed to produce homogeneity in the dust residue to ensure that any aliquots would contain representative amounts of asbestos. The procedure was believed to cause negligible breakage of fibres (but was not designed specifically to preserve the size distribution of the fibres). It was interesting, therefore, to find that there were no observable differences in the mean size distributions for fibres recovered after three, six, 12, 15 , and 18 months of exposure respectively. This apparent absence of any change in size distribution is in contrast with results from other studies ${ }^{14}$ 15 that have shown preferential clearance of the shorter fibres.

The results obtained for the masses of asbestos in the lungs are summarised in the table. The mean mass estimates are based on evaluations of 100 fibres from each of three or more lung samples. In all cases at least three such evaluations were made of the fibres longer than $5 \mu \mathrm{m}$. In most cases evaluations were made also of the fibres longer than $5 \mu \mathrm{m}$. On average, $68 \%$ of the total mass consisted of fibres longer than $5 \mu \mathrm{m}$. This factor $(68 \%)$ was used to calculate the total mass in the four cases for which data were obtained only for fibres longer than $5 \mu \mathrm{m}$.

The respirable dust concentration was kept as constant as possible for the duration of the exposure but there was some variation which shows in the time weighted average exposure concentrations given in the

Summary of the results for the amounts of amosite asbestos in the lungs of rats exposed to amosite asbestos at $0 \cdot 1 \mathrm{mg} / \mathrm{m}^{3}$

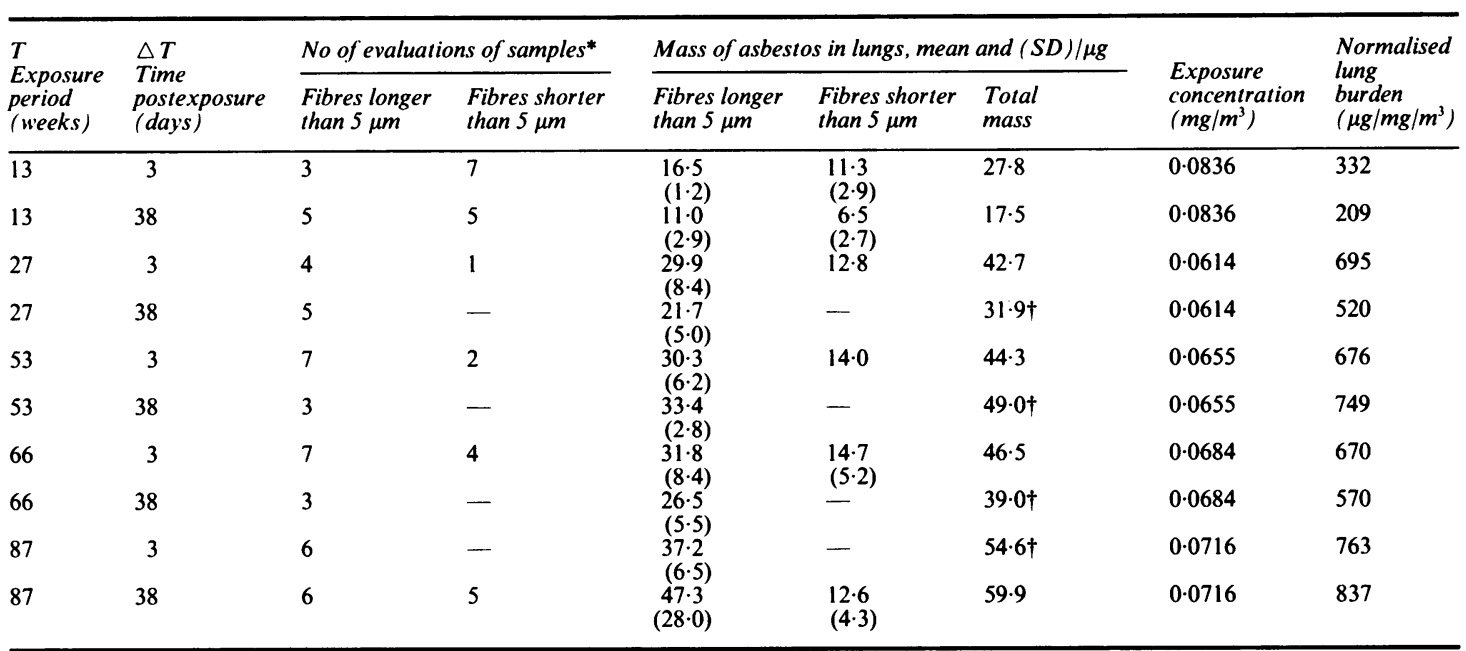

*Each evaluation consisted of counting and sizing about 100 fibres.

†Total mass estimated as $1.47 \times$ mass of fibres longer then $5 \mu \mathrm{m}$. 


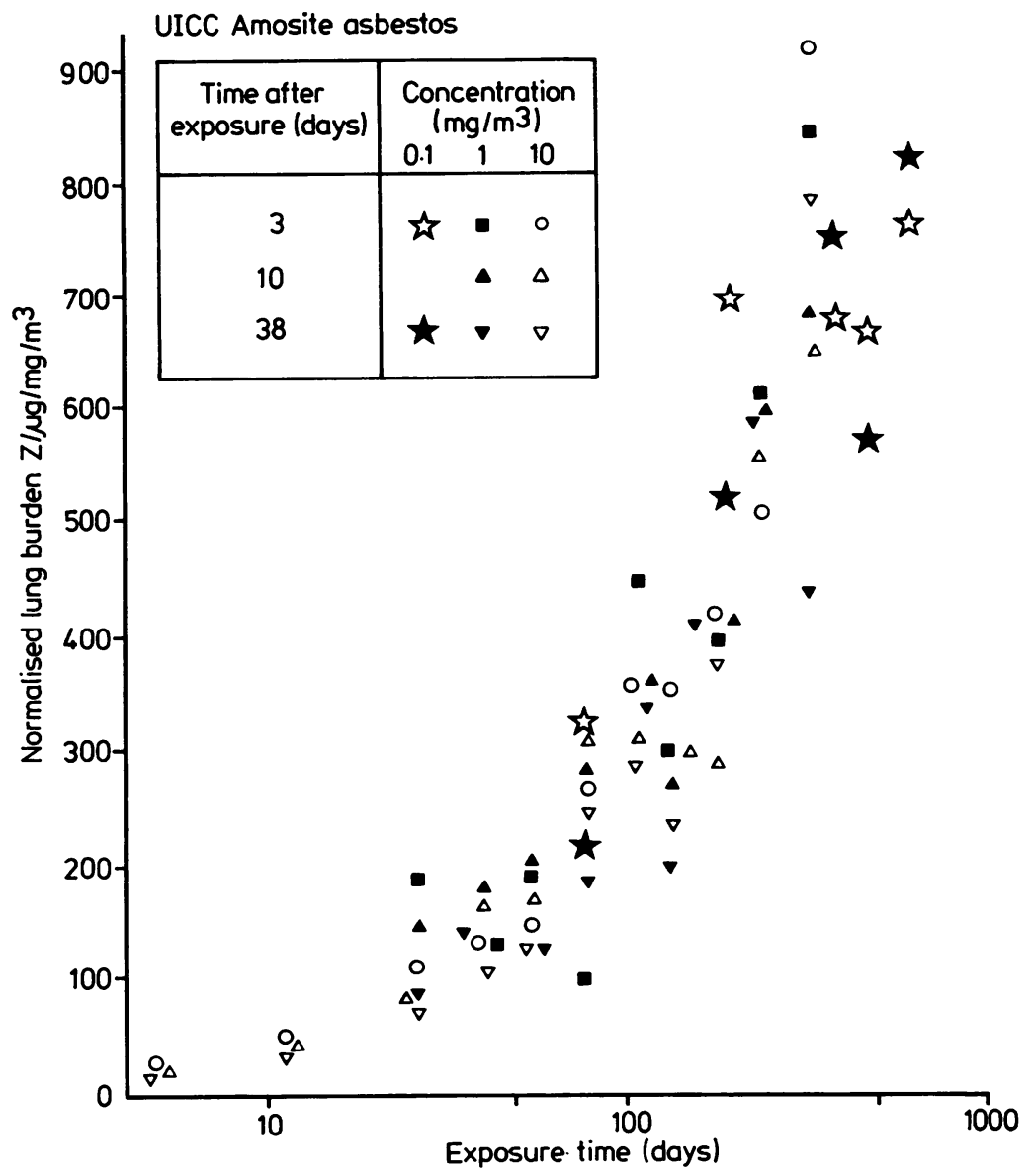

Measurements of lung burden scaled with respect to exposure concentration (Z) as a function of exposure time $(T)$ for various postexposure times $(\triangle T)$ and for three approximately constant exposure concentrations $\left(0 \cdot 1,1\right.$, and $\left.10 \mathrm{mg} / \mathrm{m}^{3}\right)$.

table for each exposure period. The standardised lung burdens $(\mathrm{Z})$ are the mean total masses of asbestos in the lung divided by the respective mean exposure concentrations. In terms of concentration by number of fibres longer than $5 \mu \mathrm{m}$, diameter less than $3 \mu \mathrm{m}$ and aspect ratio greater than 3 (as routinely used in occupational hygiene), the target exposure concentration of $0 \cdot 1 \mathrm{mg} / \mathrm{m}^{3}$ was shown (by counts on 20 samples) to correspond to approximately $15 \mathrm{ml}$.

The standardised mean lung burdens, $Z$ (from table), are plotted in the figure with results from the previous exposures at higher $\left(1\right.$ and $\left.10 \mathrm{mg} / \mathrm{m}^{3}\right)$ respirable dust concentrations. The most obvious feature of the figure is the extent to which the lung burdens scale with respect to concentration over the 100 -fold range. Results obtained for all three exposure concentrations for exposure periods up to 12 months all appear to lie within the common band of data points. Only the results obtained at the lowest concentration $(0.1 \mathrm{mg} /$ $\mathrm{m}^{3}$ ) at the longest exposure periods (beyond 12 months) show some possible indication that further accumulation might do other than follow a unique trend common to all concentrations. Here, $\mathrm{Z}$ appears to be slightly less than for the higher concentrations. The main features of the results, however, are that the lung burden continues to increase approximately pro rata with time of exposure throughout the exposure period, and that the rate of increase in actual lung burden is directly proportional to the exposure concentration.

The principal conclusion of this work is that these most recent results give further support to the kinetic model of deposition and clearance involving a sequestration compartment as being the most appropriate 
description of dosimetry for lungs exposed over a prolonged period to airborne amosite asbestos. The same form of model is also supported by recent results for other relatively insoluble minerals - for example, coal, ${ }^{16}$ quartz and titanium dioxide, ${ }^{17}$ and diesel and carbon black particulate. ${ }^{1819}$ For one mineral, chrysotile asbestos, Wagner et al have reported an appreciably different pattern of results with the lung burden reaching an equilibrium level (and ceasing to increase further) for rats exposed at a constant concentration of $10 \mathrm{mg} / \mathrm{m}^{3}$ for one year. ${ }^{20}$ This is thought to be due to the nature of the chrysotile mineral (possibly involving its dissolution in lung fluids), and this is an area that requires further investigation.

\section{References}

1 Middleton AP, Beckett ST, Davis JMG. A study of the short-term retention and clearance of inhaled asbestos, using UICC standard reference samples. In: Walton WH, ed. Inhaled particles VI. Oxford: Pergamon Press, 1977:247-57.

2 Middleton AP, Beckett ST, Davis JMG. Further observations on the short-term retention and clearance of asbestos by rats using UICC reference samples. Ann Occup Hyg 1979;22:141-52.

3 Klosterkotter W, Buneman G. Animal experiments on the elimination of inhaled dust. In: Davies CN, ed. Inhaled particles and vapours. Oxford: Pergamon Press, 1971:227-37.

4 Ferin F. Observations concerning alveolar dust clearance. Ann NY Acad Sci 1972;200:66-72.

5 Vostal JJ, Schreck RM, Lee PS, Chan TL, Soderholm SC. Deposition and clearance of diesel particles from the lung. In: Lewtas S, ed. Toxicological effects of emissions from diesel engines. New York: Elsevier Science Publishing Company, 1982:143-59.

6 Bolton RE, Vincent JH, Jones AD, Addison J, Beckett ST. An overload hypothesis for pulmonary clearance of UICC amosite fibres inhaled by rats. Br J Ind Med 1983;40:264-72.

7 Vincent JH, Johnston AM, Jones AD, Bolton RE, Addison J. Kinetics of deposition and clearance of inhaled mineral dusts during chronic exposure. Br J Ind Med 1985;42:707-15.

8 Chan TL, Lee PS, Hering WE. Pulmonary retention of inhaled diesel particles after prolonged exposure to diesel exhaust. Fundamental and Applied Toxicology 1984;4:624-31.

9 Smith TJ. Development and application of a model for estimating alveolar and interstitial dust levels. Ann Occup Hyg 1985; 29:495-516.

10 Rappaport SM. Smoothing of exposure variability at the receptor: implications for health standards. Ann Occup Hyg 1985;29: 201-14.

11 Beckett ST. The generation and evaluation of UICC asbestos in animal exposure chambers. Ann Occup Hyg 1975;18:187-98.

12 Health and Safety Executive (HSE). Asbestos-control limits, measurement of airborne dust concentrations and the assessment of control measures. London: HMSO, 1984. (Guidance note EH10.)

13 Burdett GJ, Le Guen JMM, Rood AP. Mass concentrations of airborne asbestos in the non-occupational environment-a preliminary report of UK measurements. Ann Occup Hyg 1984;28:31-8.

14 Morgan A, Holmes A. Solubility of asbestos and man-made mineral fibers in vitro and in vivo: its significance in lung disease. Environ Res 1986;39:475-84.

15 Morgan A. Effect of length on the clearance of fibres from the lung and on body formation. In: Wagner JC, ed. Biological effects of mineral fibres. Lyon: International Agency for Research on Cancer, 1980:329-35.

16 Robertson A, Bolton RE, Miller BG, et al. The effect of quartz content on the pathogenicity of coalmine dusts. In: Dodgson J, McCallum RI, eds. Inhaled particles VI. Oxford: Pergamon Press (in press).

17 Vincent JH, Jones AD, Johnston AM, McMillan CH, Bolton RE, Cowie $\mathrm{H}$. Accumulation of inhaled mineral dust in the lung and associated lymph nodes: implications to exposure and dose in occupational lung disease. Ann Occup Hyg (in press).

18 Strom KA, Chan TL, Johnston JT. Pulmonary retention of inhaled submicron particles in rats: diesel exhaust exposures and lung retention model. In: Dodgson J, McCallum RI, eds. Inhaled particles VI. Oxford: Pergamon Press (in press).

19 Strom KA, Johnston JT, Chan TL. Retention and clearance of inhaled submicron carbon black particles. Warren, Michigan: General Motors Research, 1087. (Pub GMR-5762.) (Abstract in Toxicologist 1987;7:114.

20 Wagner JC, Skidmore JW. Asbestos dust deposition and retention in rats. Ann NY Acad Sci 1965;132:77-86.

\section{Correspondence and editorials}

The British Journal of Industrial Medicine welcomes correspondence relating to any of the material appearing in the journal. Results from preliminary or small scale studies may also be published in the correspondence column if this seems appropriate. Letters should be not more than 500 words in length and contain a minimum of references. Tables and figures should be kept to an absolute minimum. Letters are accepted on the understanding that they may be subject to editorial revision and shortening.

The journal now also publishes editorials which are normally specially commissioned. The Editor welcomes suggestions regarding suitable topics; those wishing to submit an editorial, however, should do so only after discussion with the Editor. 